
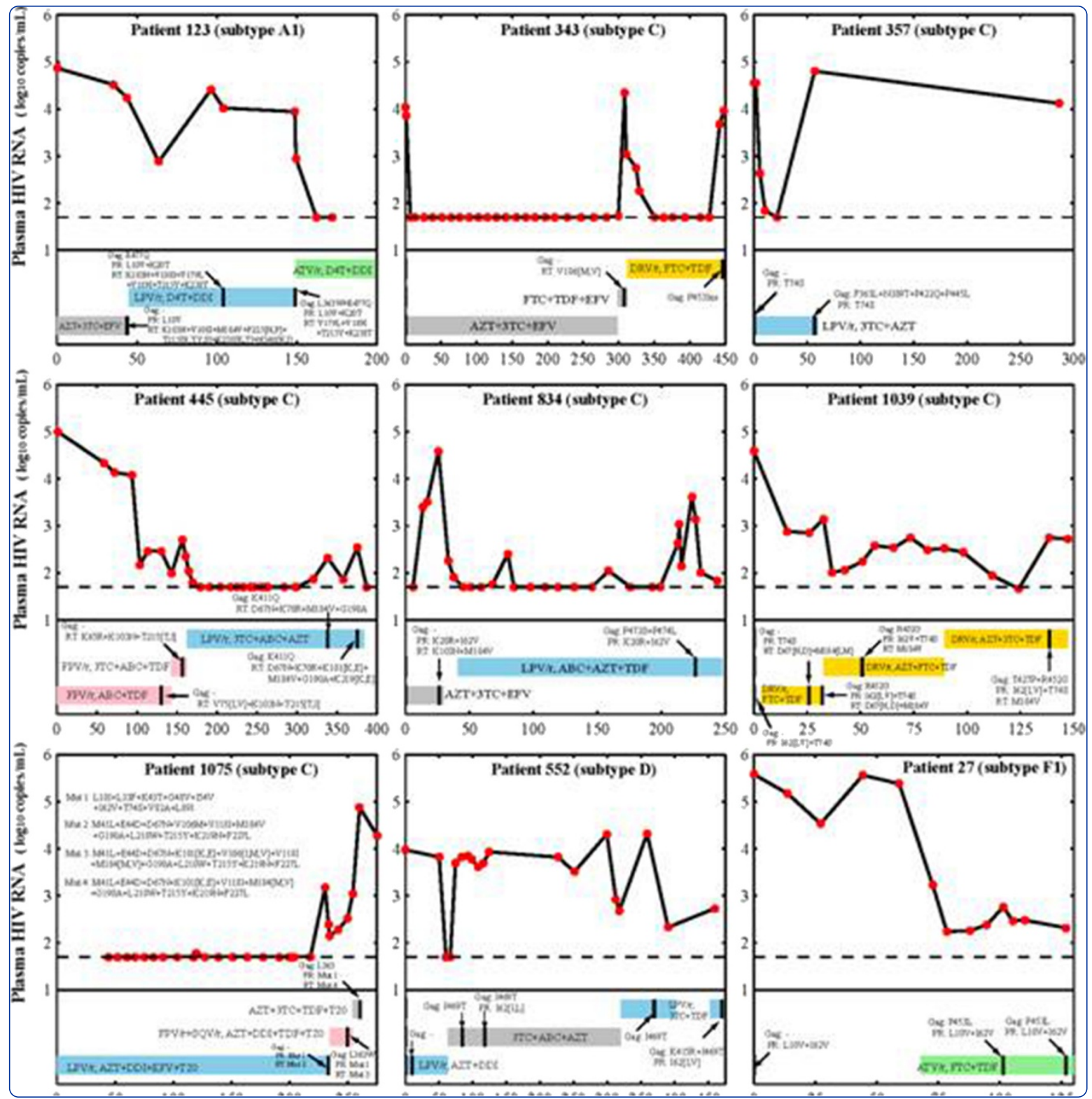

\title{
HIV-1 Gag C-terminal amino acid substitutions emerging under selective pressure of protease inhibitors in patient populations infected with different HIV-1 subtypes
}

Li et al. 


\title{
HIV-1 Gag C-terminal amino acid substitutions emerging under selective pressure of protease inhibitors in patient populations infected with different HIV-1 subtypes
}

\author{
Guangdi Li $i^{1}$, Jens Verheyen², Kristof Theys ${ }^{1}$, Supinya Piampongsant ${ }^{1}$, Kristel Van Laethem \\ and Anne-Mieke Vandamme ${ }^{1,3^{*}}$
}

\begin{abstract}
HIV-1 Gag amino acid substitutions associated with protease inhibitor (PI) treatment have mainly been reported in subtype $B$, while information on other subtypes is scarce. Using sequences from 11613 patients infected with different HIV-1 subtypes, we evaluated the prevalence of $93 \mathrm{Gag}$ amino acid substitutions and their association with genotypic PI resistance. A significant association was found for 13 Gag substitutions, including A431V in both subtype B and CRF01_AE. K415R in subtype C and S451G in subtype B were newly identified. Most Pl-associated Gag substitutions are located in the flexible C-terminal domain, revealing the key role this region plays in PI resistance.
\end{abstract}

Keywords: HIV-1 subtype, Protease, Protease inhibitor, Gag amino acid substitution, Drug resistance

\section{Findings}

An amino acid substitution is commonly defined as an amino acid change between two consecutive sequences based on longitudinal data [1,2]. Amino acid substitutions in HIV-1 protease, commonly called resistance mutations if they confer HIV-1 drug resistance, are known to emerge under selective pressure of protease inhibitors (PIs) [3]. As an alternative mechanism, HIV-1 can escape PI selective pressure by the selection of substitutions in the protease substrate Gag [1,4-7]. Such Gag substitutions arising during PI-based treatment have mostly been characterized in HIV-1 subtype B (Additional file 1: Table S1), while only a few studies have focused on non-B subtypes using small cohorts of patients (Table 1). Gag variability has been shown to impact PI susceptibility in a subtypedependent manner $[4,6]$, warranting a comprehensive analysis of PI-associated Gag substitutions across different

\footnotetext{
* Correspondence: annemie.vandamme@uzleuven.be

${ }^{1}$ Clinical and Epidemiological Virology, Rega Institute for Medical Research, Department of Microbiology and Immunology, KU Leuven - University of Leuven, Leuven, Belgium

${ }^{3}$ Centro de Malária e Outras Doenças Tropicais and Unidade de Microbiologia, Instituto de Higiene e Medicina Tropical, Universidade Nova de Lisboa, Lisboa, Portugal

Full list of author information is available at the end of the article
}

subtypes. Here, we identified novel Gag substitutions in HIV-1 non-B subtypes using longitudinal data from patients failing PI-based therapy. Moreover, we evaluated the prevalence of the newly identified and the previously reported Gag substitutions in different HIV-1 subtypes and investigated their association with genotypic PI resistance using a large sequence dataset.

We first investigated the emergence of non-B Gag substitutions during PI-based treatment in a cohort of 1068 patients followed at the University Hospital of Leuven, for which virological outcome and treatment information were available [12]. Our protocol and quality control of viral sequencing and viral load tests have been described previously [13,14]. For 69 patients infected with HIV-1 non-B subtypes and receiving PI-based treatment for at least three months, sequence information for Gag, protease and reverse transcriptase (RT) was available at baseline and at treatment failure, which was defined according to the guidelines of the European AIDS Clinical Society (EACS) (http://www.eacsociety.org/). Under drug selective pressure, 21 different substitutions at $18 \mathrm{Gag}$ positions were identified among 12 patients, of whom 11 harbored Gag substitutions in the presence of (pre-existing or simultaneously acquired) drug resistance mutations in 
Table 1 Summary of Gag amino acid substitutions in HIV-1 non-B subtypes observed during PI-based treatment

\begin{tabular}{|c|c|c|}
\hline Gag amino acid substitutions in non-B subtypes* & $\begin{array}{l}\text { Number of patients infected } \\
\text { by non-B subtypes }\end{array}$ & Reference \\
\hline A431V & $A$ or $F^{\#}(n=4)$ & {$[8]$} \\
\hline K436R, N451S & $C(n=1)$ & {$[8]$} \\
\hline L363F, A364G, A374T, I376V, M378V, R380K, K436E, G443R & $G(n=2), 01 \_A E(n=1), 02 \_A G(n=4)$ & [9] \\
\hline $\begin{array}{l}\text { V128A/T/I, Q130R, Y132F, V135M, V362I, A373T, A374T, A375T, I376AN/M, K380R, } \\
\text { S381G, N382K, E428D/Q, Q430R/G/N/I, A431V, K436R, L449I, N451S, R452K, P453I }\end{array}$ & $G(n=21)$ & [10] \\
\hline N375S, G381R & $A 1(n=2)$ & {$[2]$} \\
\hline G381S, G446E & 02_AG $(n=1)$ & {$[2]$} \\
\hline V135I, I376V, L486F & 01_AE $(n=1)$ & [2] \\
\hline $\mathrm{P} 453 \mathrm{~L} / \mathrm{T} / \mathrm{I}$ & $F^{\#}(n=61)$ & {$[11]$} \\
\hline $\begin{array}{l}\text { M138L, F363L, L363W, A374T, V374A, R387K, N389T, K411Q, K415R, G420A, } \\
\text { P422Q, T427P, P445L, S451G, R452G, P453L, P453Ins, I469T, P472S, P474L, E477Q }\end{array}$ & $\begin{array}{l}\text { A1 }(n=1), C(n=6), D(n=1), F 1(n=1) \\
J(n=1), 01 \_A E(n=1), 02 \_A G(n=1)\end{array}$ & Our study \\
\hline
\end{tabular}

*Non-B Gag substitutions reported during PI-based treatment. The substitutions are summarized based on the original publications, and for the substitutions in our study, it is given relative to the baseline sequences sampled from individual patients (see Figure 1). The substitutions also identified in subtype B are indicated in bold. Additional file 1: Table S1 summarizes the information of Gag substitutions in HIV-1 subtype B.

\#Information of HIV-1 subtype or sub-subtype was ambiguous or not available.

protease or RT (Figure 1, Additional file 1: Table S2). Gag substitution P453Ins (insertion: EPTAPP) emerged in patient 343 in the absence of PI and RTI resistance mutations. Some substitutions were from a less to a more common amino acid such as M138L. Specifically, patients failing LPV/r-based regimens developed one of the following Gag substitution patterns: L363W + E477Q, F363L + N389T + P422Q + P455L, K411Q, P472S + P474L, K415R + I469T, M138L, A374T or G420A. Patients failing DRV/r-based regimens developed Gag substitution patterns P453Ins or T427P + R452G. Patients failing an ATV/rbased regimen developed Gag substitution patterns: P453L or V374A + R387K + S451G + P453Ins. A patient failing a regimen containing FPV/r and SQV/r developed L363W. Longitudinal data from 34 PI-naïve patients infected with non-B subtypes revealed the emergence of one Gag substitution (V370A) in a single patient. Overall, when analyzing all subtypes, the proportion of PI-treated patients with Gag substitutions was much higher than that of PI-naïve patients $(17.4 \%(12 / 69)$ vs $2.9 \%(1 / 34)$, p-value $=0.037)$.

For our second analysis, we compiled a comprehensive list of $93 \mathrm{Gag}$ substitutions at 55 positions in B and non-B subtypes observed in PI-treated patients, based on literature results or our first analysis as described above (Table 1, Additional file 1: Table S1). Next, we systematically evaluated the prevalence of these variants in major HIV-1 subtypes using 10865 full-length Gag sequences retrieved from the HIV Los Alamos database (one sequence per patient) (Table 2). Sequence alignment and quality control have been described previously [15]. We found that the prevalence of 62 (66.7\%) Gag variants at 39 positions was above $1 \%$ in at least one subtype or CRF (A1, B, C, D, F1, G, CRF01_AE, CRF02_AG). Among the 55 Gag positions, only 363 and 455 were highly conserved with less than $1 \%$ overall amino acid variation in every subtype and CRF in our dataset (Figure 2A). Moreover, 77 of these 93 variants $(82.8 \%)$ were found at 42 positions located in the Gag C-terminal domain (positions: 362-500).

As treatment information of the 10865 full-length $\mathrm{gag}$ nucleotide sequences was largely lacking, our third analysis aimed to evaluate whether these $93 \mathrm{Gag}$ variants were significantly associated with genotypic PI resistance. Among the 11613 sequences pooled from the Leuven and the Los Alamos datasets (Table 2), 6645 spanned both the gag and the full-length protease regions, and were translated into amino acid sequences for our analysis. Using the drug resistance interpretation algorithms HIVdb V7.0 [16] and Rega V9.1 [17], 660 sequences were concordantly estimated to be partially or fully resistant to at least one PI, and 5657 sequences were concordantly estimated to be fully susceptible to all PIs (Additional file 1: Table S3). Sequences with discordant estimates of PI susceptibility were excluded from our analysis. Fisher's exact tests were then used to compare the amino acid prevalence between these PI-susceptible and PI-resistant datasets. Of the $93 \mathrm{Gag}$ variants, 16 at 13 amino acid positions were associated with (partial or full) PI resistance in at least one HIV-1 subtype (p-value $<0.05$, Additional file 1: Table S4). After multiple testing correction using the false discovery rate approach described in [18], $13 \mathrm{Gag}$ variants at 10 positions remained significantly PI-associated within individual subtypes (adjusted p-value $<0.05$ ), including 11 variants located in the Gag C-terminal domain (Figure 2B, Table 3). Our analysis successfully identified the known PI-associated Gag substitution A431V, strengthening the validity of our approach. As the only PI-associated Gag substitution found in more than one subtype, A431V had a high prevalence in the PI-resistant strains of subtype B (13.5\%) and CRF01_AE (18.2\%) (Table 3). Interestingly, of the 21 Gag substitutions observed in our first analysis, 

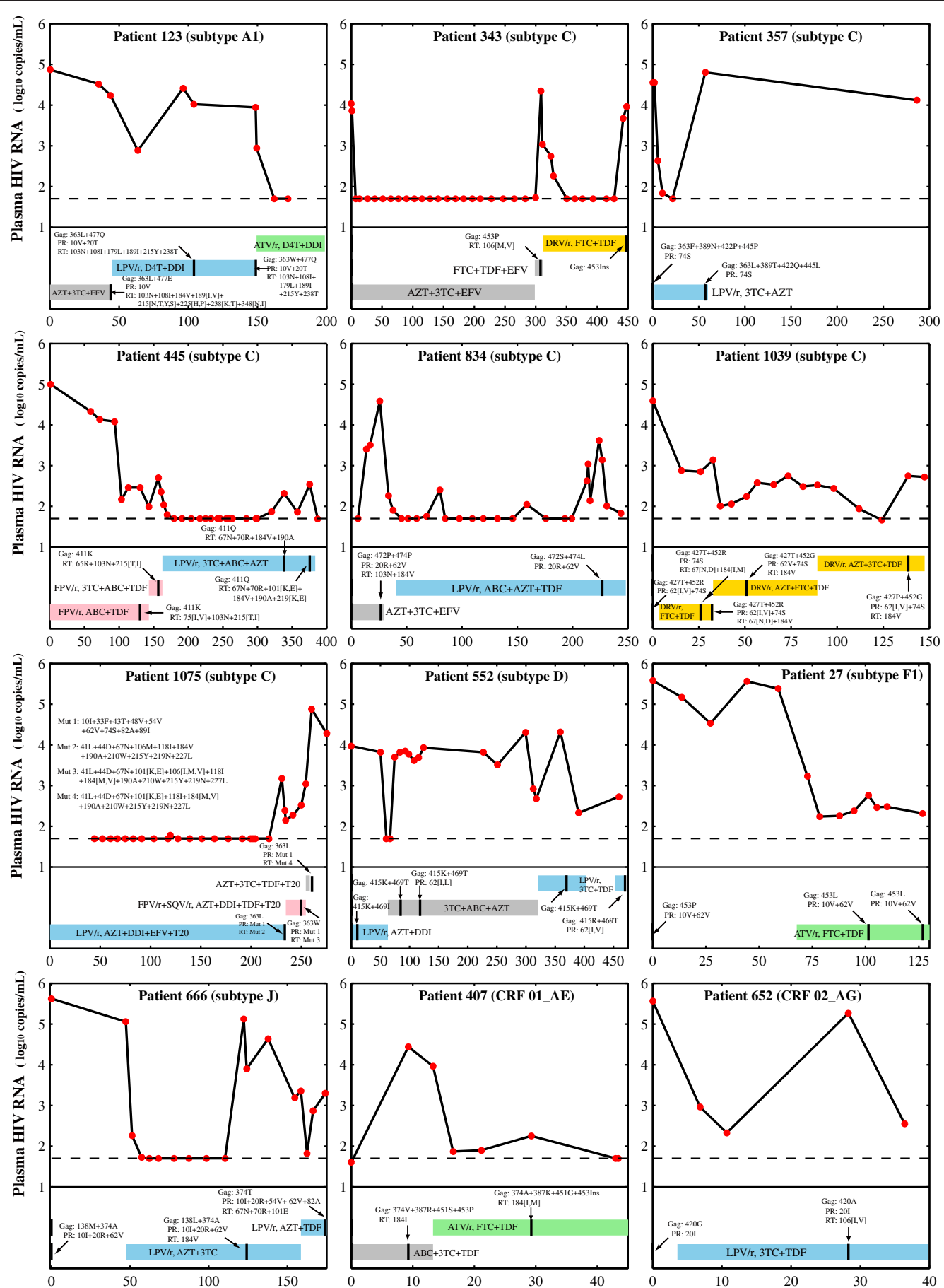

\section{Time (weeks)}

Figure 1 Gag substitutions and PI or RTI resistance mutations in 12 patients from the Leuven cohort. Each subplot shows the data of one patient regarding the viral load, the treatment period and the emerging Gag substitutions and the PI/RTI resistance mutations. X-and Y-axes indicate the time (weeks) and the level of plasma HIV RNA ( $\log _{10}$ copies $/ \mathrm{mL}$ ), respectively. For each subplot, red dots indicate the level of viral load and the dash line indicates the viral load cutoff at 50 copies per $\mathrm{mL}$. Beneath the viral load plot, each treatment period is annotated by a colored bar with vertical black lines indicating the sequence sampling time. The blue, pink, green and yellow bars show PI-based treatments containing LPV/r, FPV/r, ATV/r and DRV/r, respectively. The grey bar indicates treatments lacking Pls. Multiple substitutions or mutations are shown using the plus symbol "+". Amino acids translated from ambiguous nucleotide letters are indicated by brackets. For patient 343, the insertion EPTAPP at position P453 is annotated as P453Ins. For patient 1075, the sets of PI or RTI resistance mutation are abbreviated (Mut 1-4) and listed in the subplot. Additional file 1: Table S2 provides the full list of Gag, protease and RT substitutions in these 12 patients. 
Table 2 Summary of Leuven and Los Alamos sequence datasets

\begin{tabular}{|c|c|c|c|c|c|}
\hline \multirow[t]{2}{*}{ Subtype } & \multirow{2}{*}{$\begin{array}{l}\text { Los Alamos dataset } \\
\text { Number of Gag sequence* }\end{array}$} & \multicolumn{3}{|l|}{ Leuven dataset } & \multirow{2}{*}{$\begin{array}{l}\text { Total number } \\
\text { of patient }\end{array}$} \\
\hline & & Number of Gag sequence & Number of PI-naïve patient & Number of PI-treated patient & \\
\hline $\mathrm{A} 1$ & 1648 & 167 & 72 & 19 & 1739 \\
\hline B & 4131 & 639 & 313 & 57 & 4501 \\
\hline C & 2780 & 198 & 58 & 24 & 2862 \\
\hline D & 443 & 42 & 20 & 9 & 472 \\
\hline $\mathrm{F} 1$ & 35 & 38 & 25 & 4 & 64 \\
\hline G & 49 & 1 & 1 & 0 & 50 \\
\hline J & 3 & 8 & 1 & 2 & 6 \\
\hline 01_AE & 1714 & 72 & 45 & 5 & 1764 \\
\hline 02_AG & 62 & 139 & 71 & 22 & 155 \\
\hline Total & 10865 & 1304 & 606 & 142 & 11613 \\
\hline
\end{tabular}

*Number of Gag sequences in different HIV-1 subtypes (one sequence per patient) [15].

\section{A}

Gag positions

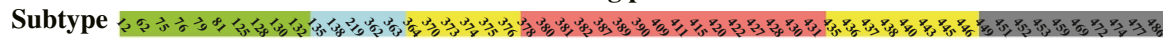

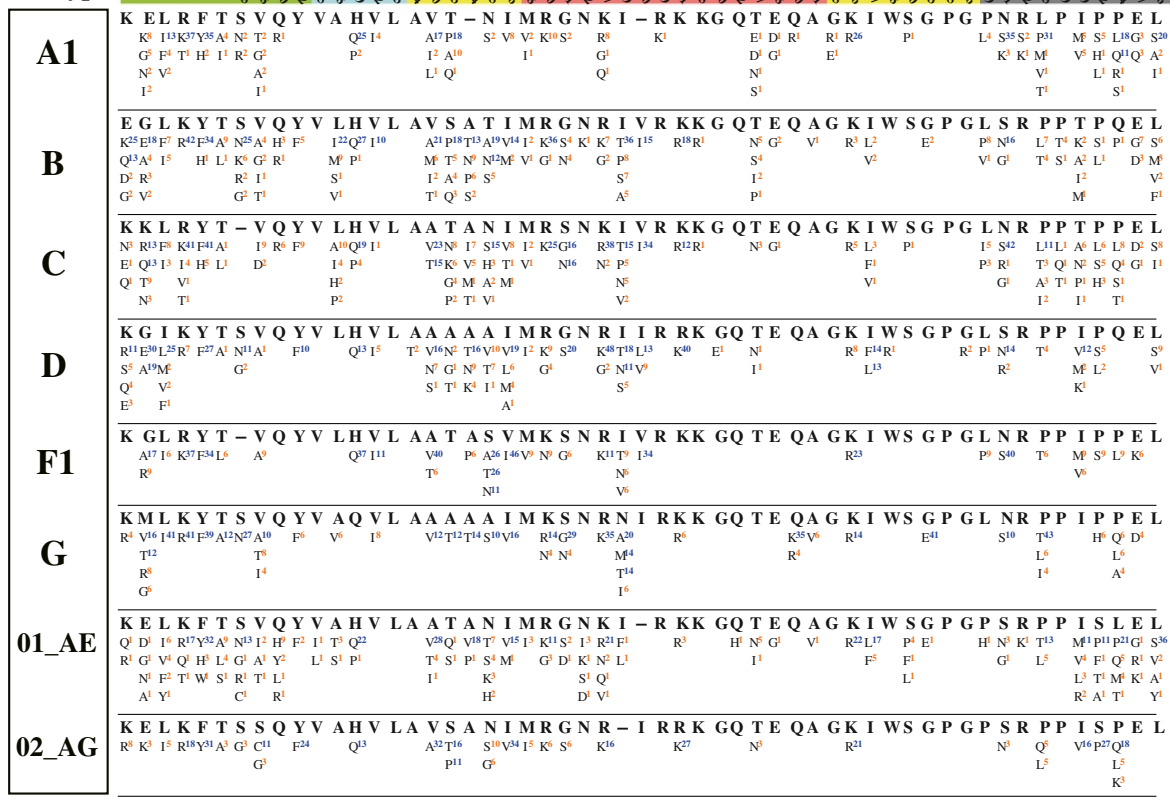

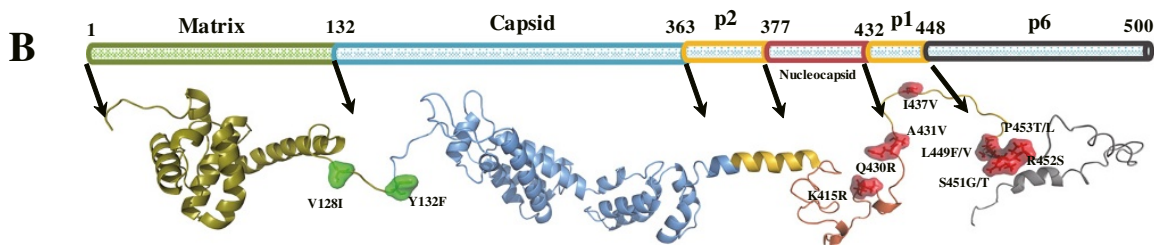

Figure 2 Prevalence of Gag amino acid variants reported in patients failing PI-based therapies and their mapping to HIV-1 protein structures. (A) Prevalence of amino acid variations at 55 Gag positions in 8 HIV-1 subtypes (A1, B, C, D, F1, G, 01_AE and 02_AG) given the Los Alamos full-length Gag sequence dataset (Table 2). Only Gag positions where amino acid substitutions have been observed during PI-based treatment are shown. For each position, the HXB2 index is shown at the top, followed by the most prevalent amino acids (bold) and amino acid variations in our sequence datasets. Amino acids with blue superscripts have prevalence above 10\% and other amino acids have orange superscripts. (B) Structural representation of Gag polyprotein and mapping of the 13 Pl-associated Gag substitutions identified in Table 3. The annotation of Gag polyproteins is shown at the top. Individual Gag protein structures are shown at the bottom. Gag substitutions are annotated and colored accordingly. Red surfaces indicate Pl-associated Gag substitutions at the Gag C-terminal domain; other substitutions are shown in green. PDB data of Gag protein structures: matrix, 1HIW; capsid, 3NTE; p2, 1U57; nucleocapsid, 2M3Z; p6, 2C55. Visualization software: PyMOL V1.5 (http://www.pymol.org/). 
Table 3 Prevalence of PI-associated Gag substitutions in individual HIV-1 subtypes

\begin{tabular}{|c|c|c|c|c|c|}
\hline \multirow{2}{*}{$\begin{array}{l}\text { Gag } \\
\text { substitutions* }\end{array}$} & \multirow[t]{2}{*}{ Subtype } & \multicolumn{2}{|l|}{ Prevalence $^{\#}$} & \multirow[t]{2}{*}{ P-value } & \multirow{2}{*}{$\begin{array}{l}\text { Adjustec } \\
\text { p-value }\end{array}$} \\
\hline & & PI-resistant dataset & PI-susceptible dataset & & \\
\hline V128I & B & $5.8 \%(7 / 121 \&)$ & $0.9 \%(6 / 638)$ & 0.002 & 0.024 \\
\hline Y132F & B & $10.7 \%(13 / 122)$ & $3.4 \%(22 / 639)$ & 0.004 & 0.035 \\
\hline K415R & C & $2.5 \%(3 / 119)$ & $0.0 \%(0 / 1727)$ & $<0.0001$ & 0.012 \\
\hline Q430R & C & $2.5 \%(3 / 119)$ & $0.1 \%(1 / 1727)$ & 0.003 & 0.046 \\
\hline \multirow[t]{2}{*}{ A431V } & B & $13.5 \%(23 / 170)$ & $0.1 \%(1 / 787)$ & $<0.0001$ & $<0.0001$ \\
\hline & 01_AE & $18.2 \%(4 / 22)$ & $0.7 \%(8 / 1111)$ & $<0.0001$ & 0.007 \\
\hline $1437 \mathrm{~V}$ & B & $8.9 \%(15 / 168)$ & $1.7 \%(13 / 784)$ & $<0.0001$ & $<0.0001$ \\
\hline L449F & B & $5.6 \%(21 / 377)$ & $0.5 \%(7 / 1352)$ & $<0.0001$ & $<0.0001$ \\
\hline L449V & B & $4.8 \%(18 / 377)$ & $0.9 \%(12 / 1352)$ & $<0.0001$ & $<0.0001$ \\
\hline S451G & B & $3.4 \%(13 / 378)$ & $1.3 \%(17 / 1348)$ & 0.008 & 0.041 \\
\hline S451T & B & $2.1 \%(8 / 378)$ & $0.0 \%(0 / 1348)$ & $<0.0001$ & $<0.0001$ \\
\hline R452S & B & $3.4 \%(13 / 384)$ & $0.3 \%(4 / 1374)$ & $<0.0001$ & $<0.0001$ \\
\hline P453T & C & $21.8 \%(26 / 119)$ & $3.1 \%(53 / 1722)$ & $<0.0001$ & $<0.0001$ \\
\hline P453L & B & $18.5 \%(71 / 384)$ & $7.1 \%(99 / 1399)$ & $<0.0001$ & $<0.0001$ \\
\hline
\end{tabular}

*A list of Gag substitutions whose prevalence differs significantly between sequences estimated to be (fully or partially) PI-resistant and sequences estimated to be PI-susceptible (see full reports in Additional file 1: Table S4). The substitutions are indicated relative to the consensus amino acids from individual subtypes [15]. One-tailed Fisher's exact tests were performed, and p-values were adjusted using multiple testing correction via the false discovery rate (FDR) approach [18]. ${ }^{\#}$ Statistical analyses were only performed on individual subtype (B, C, G, 01_AE) datasets, which contained more than 10 (partially or fully) PI-resistant sequences. Additional file 1: Table S3 summarizes the subtype distribution of PI-resistant and PI-susceptible sequence datasets.

\&: The numerator indicates the number of sequences for which the corresponding Gag position is covered; the denominator indicates the number of sequences displaying the respective amino acid substitutions.

K415R and S451G were newly identified to be significantly associated with genotypic PI resistance in subtypes $\mathrm{C}$ and $B$ respectively, suggesting a possible involvement in PI-resistance.

To our knowledge, this study presents the first largescale sequence analysis to establish statistical significance of PI-associated Gag substitutions in HIV-1 non-B subtypes. Our longitudinal analysis of a clinical cohort of patients failing PI-based therapy confirmed that PItreated patients developed more Gag substitutions than PI-naïve patients. The majority of these Gag substitutions emerged in the context of pre-existing or simultaneously acquired PI or RTI resistance mutations, confirming the important role of the known resistance mutations, while in some patients Gag substitutions emerged in the absence of resistance mutations (Figure 1, Additional file 1: Table S2). Such Gag substitutions may therefore contribute to the virological failure of PI-based treatments. Based on two widely used genotypic interpretation algorithms, our comparative analysis found that only 13 (13.8\%) of the 93 Gag substitutions emerging under PI selective pressure were significantly associated with genotypic PI resistance (Table 3). Particularly, the novel Gag substitutions K415R and S451G were identified in both our longitudinal and cross-sectional sequence analyses. This suggests that they may play a role in viral escape from PI selective pressure, partially contributing to the observed virological failure. Since virological outcome and treatment information is lacking for most sequences extracted from the HIV Los Alamos database, this limits our analysis to address the clinical impact of the newly identified substitutions with large-scale data. Using small cohorts, previous studies suggested that different subtypes may develop different Gag substitutions $[6,19,20]$. We confirmed this hypothesis since only 9 of the $58 \mathrm{Gag}$ substitutions reported in non-B subtypes (Table 1) were also observed in subtype B (Additional file 1: Table S1). Among non-B Gag substitutions, 4 were significantly associated with genotypic PI resistance, of which only A431V was PI-associated in subtype B as well (Table 3). However, further evaluations on subtypes A2, D, F2, J, $\mathrm{K}$ and other CRFs are still needed due to the restriction of our study to particular subtypes. Interestingly, a predominant presence of PI-associated Gag substitutions at the flexible C-terminal domain of Gag (Figure 2B) leads us to suggest the hypothesis that PI-associated Gag substitutions tend to emerge in the structural flexible regions. These Gag substitutions can emerge along with protease drug resistance mutations as shown in our longitudinal sequence analysis (Figure 1, Additional file 1: Table S2) and previous studies [21,22]. Future studies are still needed to investigate the significance of coevolution between Gag substitutions and protease resistance mutations.

Overall, our findings showed different PI-associated substitutions in the Gag C-terminal domain across 
different subtypes, providing a roadmap to elucidate the role of Gag amino acid substitutions in the development of PI resistance.

Our Leuven sequences with associated information are available through Euresist (http://www.euresist.org). The protocol and this consent procedure have been approved by the Ethical Committee UZ Leuven (reference ML-8627, approval B322201316521 S52637). Our toolbox designed for visualizing the longitudinal data in Figure 1 is freely available in Additional file 2: Toolbox S1.

\section{Additional files}

Additional file 1: Table S1. Summary of HIV-1 subtype B Gag amino acid substitutions observed during PI-based treatment. Table S2. Summary of Gag, protease and RT amino acid substitutions in the Leuven cohort. Table S3. Summary of PI-resistant and Pl-susceptible sequence datasets. Table S4. Prevalence of Gag amino acid variants in individual HIV-1 subtypes.

Additional file 2: Software. Toolbox S1: Our Matlab toolbox designed for visualizing longitudinal data of viral load, treatment period and sampling time.

\section{Abbreviations}

CRF: Circulating recombinant form; EACS: European AIDS clinical society; FDR: False discovery rate; PDB: Protein data bank; PR: Protease; PI: Protease inhibitor; RT: Reverse transcriptase; RTI: Reverse transcriptase inhibitor.

\section{Competing interests}

The authors declare that they have no competing interests.

\section{Authors' contributions}

GL, KT and AV designed the study and drafted the manuscript, KVL and JV conducted viral sequencing and viral load tests. GL, SP and KVL performed the mutation analysis. All authors contributed to the critical revision of the study and provided final approval of the version to be published.

\section{Acknowledgements}

We thank Fossie Ferreira, Lore Vinken, Yoeri Schrooten, Jasper Edgar Neggers, Nádia Conceição Neto, Liana Eleni Kafetzopoulou, Dan Clements and Jurgen Vercauteren for technical assistance and valuable contributions to the analysis. This work was supported by the AIDS Reference Laboratory of Leuven that receives support from the Belgian Ministry of Social Affairs through a fund within the Health Insurance System; the Fonds voor Wetenschappelijk Onderzoek - Flanders (FWO) [PDO/11 to K.T., G.0692.14] and the European Community's Seventh Framework Programme (FP7/2007-2013) under the project "Collaborative HIV and Anti-HIV Drug Resistance Network (CHAIN)" [223131].

\section{Author details}

${ }^{1}$ Clinical and Epidemiological Virology, Rega Institute for Medical Research, Department of Microbiology and Immunology, KU Leuven - University of Leuven, Leuven, Belgium. ${ }^{2}$ Institute of Virology, University Hospital, University Duisburg-Essen, Essen, Germany. ${ }^{3}$ Centro de Malária e Outras Doenças Tropicais and Unidade de Microbiologia, Instituto de Higiene e Medicina Tropical, Universidade Nova de Lisboa, Lisboa, Portugal.

Received: 19 May 2014 Accepted: 1 September 2014 Published online: 25 September 2014

\section{References}

1. Larrouy L, Vivot A, Charpentier C, Benard A, Visseaux B, Damond F, Matheron S, Chene G, Brun-Vezinet F, Descamps D, Cohort ACH-: Impact of gag genetic determinants on virological outcome to boosted lopinavir-containing regimen in HIV-2-infected patients. AIDS 2013, 27:69-80.
2. Larrouy L, Chazallon C, Landman R, Capitant C, Peytavin G, Collin G, Charpentier C, Storto A, Pialoux G, Katlama C, Girard PM, Yeni P, Aboulker JP, Brun-Vezinet F, Descamps D, Group AS: Gag mutations can impact virological response to dual-boosted protease inhibitor combinations in antiretroviral-naive HIV-infected patients. Antimicrob Agents Chemother 2010, 54:2910-2919.

3. Wensing AM, van Maarseveen NM, Nijhuis M: Fifteen years of HIV Protease Inhibitors: raising the barrier to resistance. Antiviral Res 2010, 85:59-74.

4. Fun A, Wensing AM, Verheyen J, Nijhuis M: Human Immunodeficiency Virus Gag and protease: partners in resistance. Retrovirology 2012, 9:63.

5. Larrouy L, Charpentier C, Landman R, Capitant C, Chazallon C, Yeni P, Peytavin G, Damond F, Brun-Vezinet F, Descamps D, group As: Dynamics of gag-pol minority viral populations in naive HIV-1-infected patients failing protease inhibitor regimen. AIDS 2011, 25:2143-2148.

6. Gupta RK, Kohli A, McCormick AL, Towers GJ, Pillay D, Parry CM: Full-length HIV-1 Gag determines protease inhibitor susceptibility within in vitro assays. AIDS 2010, 24:1651-1655.

7. Knops E, Kemper I, Schulter E, Pfister H, Kaiser R, Verheyen J: The evolution of protease mutation $76 \mathrm{~V}$ is associated with protease mutation $46 \mathrm{I}$ and gag mutation 431 V. AIDS 2010, 24:779-781.

8. Bally F, Martinez R, Peters S, Sudre P, Telenti A: Polymorphism of HIV type 1 gag p7/p1 and p1/p6 cleavage sites: clinical significance and implications for resistance to protease inhibitors. AIDS Res Hum Retroviruses 2000, 16:1209-1213.

9. Ghosn J, Delaugerre C, Flandre P, Galimand J, Cohen-Codar I, Raffi F, Delfraissy JF, Rouzioux C, Chaix ML: Polymorphism in Gag gene cleavage sites of HIV-1 non-B subtype and virological outcome of a first-line lopinavir/ritonavir single drug regimen. PLoS One 2011, 6:e24798.

10. Knops E, Daumer M, Awerkiew S, Kartashev V, Schulter E, Kutsev S, Brakier-Gingras L, Kaiser R, Pfister H, Verheyen J: Evolution of protease inhibitor resistance in the gag and pol genes of HIV subtype $\mathrm{G}$ isolates. J Antimicrob Chemother 2010, 65:1472-1476.

11. Rossi AH, Rocco CA, Mangano A, Sen L, Aulicino PC: Sequence variability in p6 gag protein and gag/pol coevolution in human immunodeficiency type 1 subtype F genomes. AIDS Res Hum Retroviruses 2013, 29:1056-1060.

12. Libin P, Beheydt G, Deforche K, Imbrechts S, Ferreira F, Van Laethem K, Theys K, Carvalho AP, Cavaco-Silva J, Lapadula G, Torti C, Assel M, Wesner S, Snoeck J, Ruelle J, De Bel A, Lacor P, De Munter P, Van Wijngaerden E, Zazzi M, Kaiser R, Ayouba A, Peeters M, de Oliveira T, Alcantara LC, Grossman Z, Sloot P, Otelea D, Paraschiv S, Boucher C, Camacho RJ, Vandamme AM: RegaDB: community-driven data management and analysis for infectious diseases. Bioinformatics 2013, 29:1477-1480.

13. Van Laethem K, Schrooten Y, Dedecker S, Van Heeswijck L, Deforche K, Van Wijngaerden E, Van Ranst M, Vandamme AM: A genotypic assay for the amplification and sequencing of gag and protease from diverse human immunodeficiency virus type 1 group M subtypes. J Virol Methods 2006, 132:181-186.

14. Maes B, Schrooten Y, Snoeck J, Derdelinckx I, Van Ranst M, Vandamme AM, Van Laethem K: Performance of ViroSeq HIV-1 Genotyping System in routine practice at a Belgian clinical laboratory. J Virol Methods 2004, 119:45-49.

15. Li G, Verheyen J, Rhee SY, Voet A, Vandamme AM, Theys K: Functional conservation of HIV-1 gag: implications for rational drug design. Retrovirology 2013, 10:126.

16. Liu TF, Shafer RW: Web resources for HIV type 1 genotypic-resistance test interpretation. Clinical infectious diseases 2006, 42:1608-1618.

17. Van Laethem K, De Luca A, Antinori A, Cingolani A, Perna CF, Vandamme AM: A genotypic drug resistance interpretation algorithm that significantly predicts therapy response in HIV-1-infected patients. Antivir Ther 2002, 7:123-129.

18. Storey JD: A direct approach to false discovery rates. Journal of the Royal Statistical Society: Series B (Statistical Methodology) 2002, 64:479-498.

19. de Oliveira T, Engelbrecht S, Janse van Rensburg E, Gordon M, Bishop K, zur Megede J, Barnett SW, Cassol S: Variability at human immunodeficiency virus type 1 subtype $C$ protease cleavage sites: an indication of viral fitness? J Virol 2003, 77:9422-9430.

20. Martins AN, Arruda MB, Pires AF, Tanuri A, Brindeiro RM: Accumulation of P(T/S) AP late domain duplications in HIV type 1 subtypes $B, C$, and $F$ derived from individuals failing ARV therapy and ARV drug-naive patients. AIDS Res Hum Retroviruses 2011, 27:687-692.

21. Mo H, Parkin N, Stewart KD, Lu L, Dekhtyar T, Kempf DJ, Molla A: Identification and structural characterization of I84C and I84A mutations that are 
associated with high-level resistance to human immunodeficiency virus protease inhibitors and impair viral replication. Antimicrob Agents Chemother 2007, 51:732-735.

22. Kolli M, Stawiski E, Chappey C, Schiffer CA: Human immunodeficiency virus type 1 protease-correlated cleavage site mutations enhance inhibitor resistance. J Virol 2009, 83:11027-11042.

doi:10.1186/s12977-014-0079-7

Cite this article as: Li et al:: HIV-1 Gag C-terminal amino acid substitutions emerging under selective pressure of protease inhibitors in patient populations infected with different HIV-1 subtypes. Retrovirology 2014 11:79.

\section{Submit your next manuscript to BioMed Central and take full advantage of:}

- Convenient online submission

- Thorough peer review

- No space constraints or color figure charges

- Immediate publication on acceptance

- Inclusion in PubMed, CAS, Scopus and Google Scholar

- Research which is freely available for redistribution 